Fizika Nizkikh Temperatur, 1998, v. 24, No 5, p. 498-500

Letters to the Editor

\title{
Thermal transport through Luttinger liquid constriction
}

\author{
I. V. Krive \\ B. I. Verkin Institute for Low Temperature Physics and Engineering, National Academy of Sciences of Ukraine, \\ 47, Lenin Ave., 310164, Kharkov, Ukraine \\ E-mail: krive@ilt.kharkov.ua \\ Received February 23, 1998
}

\begin{abstract}
The heat transport through one-dimensional quantum wire is considered in the frameworks of inhomogeneous Tomonaga-Luttinger liquid model. It is shown that even for perfect (impurity free) wire thermal transport is suppressed due to multiple scattering of plasmons on the boundaries which connect quantum wire to the leads of noninteracting electrons. In the presence of impurity inside the Luttinger liquid constriction resonant enhancement of thermal conductivity at certain conditions is predicted.
\end{abstract}

PACS: 71.10.Pm

Recently Kane and Fisher [1] studied the heat transport in Luttinger liquid (LL). They claimed that: (i) in pure LL thermal conductance $K(T)$ does not depend on electron-electron interaction and it coincides with the one of Fermi liquid (FL) $K_{0}(T)=\left(\pi^{2} / 3\right) T / h(h$ is the Planck constant, $T$ is the temperature); (ii) in the presence of single impurity $K(T) \propto T^{3}$ at low temperatures and for strong repulsive interaction $g<1 / 2$ ( $g$ is the correlation parameter of LL).

Both above results were obtained for infinitely long LL. In this case it is known [2] that dc electrical conductance $G(T)$ (formally defined as response function to the change in chemical potential) is renormalized by interaction. For pure LL $G_{0}=g e^{2} / h$ and in the presence of single impurity $G(T) \propto T^{2 / g-2}$. So the authors of Ref. 1 concluded that the ratio of thermal to electrical conductance $L_{g}=K / T G$ is modified by interaction. In FL this quantity, known as the Lorentz number, is universal $L_{0}=\left(\pi^{2} / 3\right)\left(k_{B} / e\right)^{2}\left(k_{B}\right.$ is the Boltzman constant). In infinite perfect LL wire $L_{g}=L_{0} / g$ and in the presence of impurity Lorentz «number» diverges as $T \rightarrow 0$ for $g<1 / 2$ [1].

The predictions obtained for homogeneous infinite LL can not be applied directly to the realistic situation when LL wire is connected to the reservoirs of noninteracting electrons (source and drain leads). In this case it was proved [3] that in the absence of electron backscattering dc electrical conductance is not renormalized by interaction.
The purpose of the present communication is to reconsider Kane-Fisher problem [1] for realistic experimental setup. We will study thermal transport through LL constriction in the frameworks of inhomogeneous Tomonaga-Luttinger liquid (ITLL) model [3]. For simplicity we consider here only the case of spinless electrons.

If electrons do not backscattered by inhomogenities, the entropy is totally carried by plasmons. The simplest way to visualize charge and heat transport in repulsively interacting electron system is to consider the motion of $1 D$ Wigner solid (WS) through the constriction. At zero temperature the rigid shift of WS results in interaction independent conductance $e^{2} / h$. It is evident that at finite temperatures thermally activated sound waves in WS (plasmons) can not affect electrical transport through perfect LL constriction. However the scattering of plasmons on the interfaces LL-FL will suppress heat transport.

The heat transport associated with plasmons can be expressed in terms of transmission probability, $T_{t}(\varepsilon)$, of plasmons through LL constriction. The corresponding formula for thermal conductance is readily derived in Landauer-Buttiker formalism for transport coefficients (see e.g. [4])

$$
K(T)=\frac{1}{T h} \int_{0}^{\infty} d \varepsilon \varepsilon^{2}\left(\frac{-\partial f_{B}}{\partial \varepsilon}\right) T_{t}(\varepsilon)
$$


where $f_{B}(\varepsilon)=\left(e^{\varepsilon / T}-1\right)^{-1}$ is the distribution function of plasmons. In ITLL model it is assumed [3] that the transition from interacting to noninteracting electrons is smooth (the characteristic length $\lambda_{F}<<\xi<<L$, where $\lambda_{F}$ is the Fermi wavelength and $L$ is the length of LL wire). Therefore Eq. (1) determines exact thermal conductance for perfect (impurity free) LL when Lagrangian of LL is quadratic in terms of boson variables (the leads are modelled by $1 D$ noninteracting electrons which correspond to $g=1 \mathrm{LL}$ ). In the presence of electron backscattering inside LL constriction Eq. (1) for $g<1 / 2$ determines the main contribution to heat transport at low temperatures.

So our problem is reduced to the calculation of plasmon transmission coefficient. Here we consider the special case when the scattering potential is placed exactly in the middle of LL wire (in this case one could expect the enhancement of heat transport due to resonant tunneling of plasmons through the impurity).

Backscattering of electrons causes the appearence of nonlinear local term in bosonic form of LL Lagrangian $\delta L=-V_{p} \delta(x) \cos \varphi$. For our problem this term describes the scattering of plasmons, $\varphi<<1$, on $\delta$-function potential and we immediately find the desired trasmission coefficient for infinite LL wire $T_{t}(\varepsilon)=\varepsilon^{2} /\left(\varepsilon^{2}+\varepsilon_{0}^{2}\right)$, where $\varepsilon_{0}=$ $=2 \pi g V_{p} / \hbar$. The heat conductance Eq. (1) reads

$$
K_{\delta}(T)=K_{0}\left[1+3 z+6 z^{2}-6 z^{3} \psi^{\prime}(z)\right], z=\frac{g V_{p}}{T} .
$$

Here $\psi(z)=d \ln \Gamma(z) / d z, \Gamma(z)$ is the gamma-function. At low temperatures, $T \ll g V_{p}$, Eq. (2) reproduces $T^{3}$-behavior of heat conductance found in Ref. [1].

To calculate trasmission probability of plasmons through LL constriction we should additionally take into account the scattering of plasmons on LL-FL boundaries. For adiabatic contact (ITLL model) it can be done by matching the wave functions of plasmons at boundaries. The straightforward calculations yield

$$
\begin{gathered}
T_{t}^{g}(\varepsilon)=\left(\frac{\varepsilon}{\varepsilon_{0}}\right)^{2}\left\{\left[g_{-}-g_{+}\left(\cos \frac{\varepsilon}{\Delta}+\frac{\varepsilon}{\varepsilon_{0}} \sin \frac{\varepsilon}{\Delta}\right)\right]^{2}+\right. \\
\left.+\left(\sin \frac{\varepsilon}{\Delta}+\frac{\varepsilon}{\varepsilon_{0}} \cos \frac{\varepsilon}{\Delta}\right)^{2}\right\}^{-1},
\end{gathered}
$$

where $\Delta=\hbar s / L, g_{ \pm}=1 / 2\left(g^{-1} \pm g\right), g=s / v_{F}, s$ is the plasmon velocity.

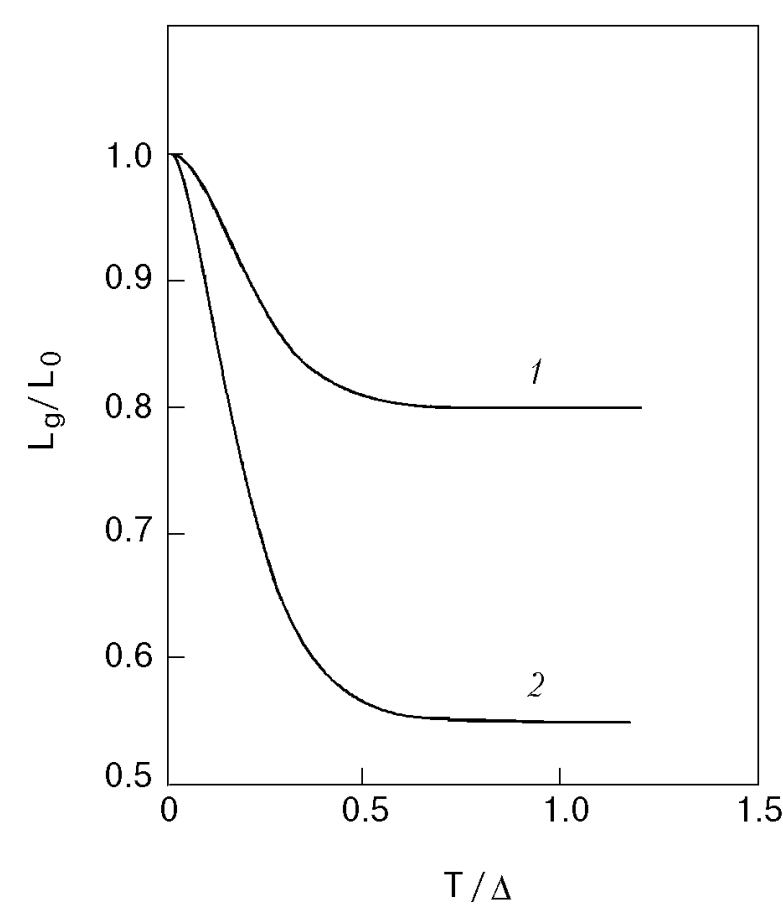

Fig. 1. The dependence of Lorentz «number» of perfect LL constriction on temperature at different values of $g: 0,5(1)$ and $0,3(2)$.

At first we consider the case of pure LL constriction $\left(\varepsilon_{0} \rightarrow 0\right)$. In this limit the transmission coefficient $T_{t}(\varepsilon)=\left[\cos ^{2}(\varepsilon / \Delta)+g_{+}^{2} \sin ^{2}(\varepsilon / \Delta)\right]^{-1}$ is a periodic function of plasmon energy with the period $\varepsilon_{n}=n \pi \Delta$ ( $n$ is integer). This property is the manifestation of quantization of plasmons in LL constriction [5]. Plasmon transport through LL wire is resonant at energies in the vicinity of $\varepsilon_{n}\left(T_{t}\left(\varepsilon_{n}\right)=1\right)$ and is suppressed (strongly suppressed $T_{t} \ll 1$ for strong interaction $g<<1$ ) at off-resonance energies.

At low temperatures $T<<\Delta$ thermal conductance $K(T)$ is determined by the contribution of long wavelength plasmons ( $n=0$ resonance) to integral Eq. (1). Low energies of plasmons correspond to the region of noninteracting electrons and therefore thermal transport is not affected by interaction [1]. Notice however that for long wires the corresponding temperature interval is contracted to a point. On contrary at temperatures $T>\Delta$ heat conductance is strongly (for $g<<1$ ) renormalized by interaction $K(T) / K_{0} \simeq 2 g<<1$. The dependence of Lorentz «number» on temperature for pure LL constriction is shown on Fig. 1. It clearly demonstrates the violation of Wiedemann-Franz law for LL wires (the effect is pronounced for the case of strong interaction $g<<1$ ).

Now we proceed to the transport properties of LL wire with impurity. Electrical conductance for the problem under study was calculated in Ref. [6]. 
As it was expected from physical considerations [2], conductance of finite LL wire at $T<<$ is temperature independent $G \simeq\left(e^{2} / h\right)\left(\Delta / g V_{p}\right)^{2 / g-2}$ and the infinite LL-like behavior [2] is restored only at $T>>\Delta$. The behavior of thermal conductance can be found from Eqs. (1), (3). At low temperatures one can neglect the quantization of plasmons and $T^{3}$ dependence of infinite LL wire, Eq. (2), $K(T) \simeq K_{0}(T)\left(T / g V_{p}\right)^{2}$ could be expected. However in exactly the same manner as for electrical conductance, the factor $\left(T / g V_{p}\right)^{2}$ caused by interaction effects is replaced by $\left(\Delta / g V_{p}\right)^{2}$. With the increase of temperature the quantization of plasmons comes into play. For our geometry when impurity is placed in the middle of LL wire resonant tunneling of plasmons through impurity occurs and thermal conductance at $T>>\Delta$ attains its maximum for the perfect LL constriction value $K(T) \simeq 2 g K_{0}(T)(g<<1)$. Therefore the Lorentz «number» of LL constriction is inverse function of temperature as it was predicted in Ref. 1. For the resonance case studied above the «high» temperature $(T>\Delta)$ behavior of $L_{g}$ is determined by the temperature dependence of conductance of LL constriction [6] and hence $L_{g} / L_{0} \propto g\left(g V_{p} / T\right)^{2 / g-2}$. Besides unlike for infinite homogeneous LL in a finite LL wire Lorentz number for $g<1 / 2$ saturates at value $L_{g} \simeq L_{0}\left(g V_{p} / \Delta\right)^{2 / g-4}$ for $T \rightarrow 0$.

When the above material was prepared for publication the author got to know that the thermal transport in quantum wires was considered recently in Ref. 7. Our results coincide with the ones of the cited paper in the places where two investigations are overlapped (pure LL constriction).

The author wish to thank V. Kravtsov, R. Shekhter and $\mathrm{Yu} \mathrm{Lu}$ for fruitfull discussions. This work was partly supported by the Royal Swedish Academy of Science (KVA) and the Natural Science Research Council (NFR). The hospitality of the Abdus Salam International Center for Theoretical Physics is greatfully acknowledged.

1. C. L. Kane and M. P. A. Fisher, Phys. Rev. Lett. 76, 3192 (1996).

2. C. L. Kane and M. P. A. Fisher, Phys. Rev. Lett. 68, 1220 (1992); Phys. Rev. B46, 15233 (1992).

3. D. L. Maslov and M. Stone, Phys. Rev. B52, R5539 (1995);

V. V. Ponomarenko, Phys. Rev. B52, R8666 (1995); I. Safi, and H. J. Schulz, Phys. Rev. B52, R17040 (1995).

4. U. Sivan and Y. Imry, Phys. Rev, B33, 551 (1986).

5. Y. V. Nazarov, A. A. Odintsov, and D. V. Averin, Europhys. Lett, 37, 213 (1997).

6. A. Furusaki and N. Nagaosa, Phys. Rev. B54, R5239 (1996).

7. R. Fazio, F. W. J. Hekking, and D. E. Khmelnitskii, «Anomalous Thermal Transport in Quntum Wires» (unpublished). 